\title{
Modelling and optimisation of the operation of a radiant warmer
}

\author{
Anna M. Fic ${ }^{1}$, Derek B. Ingham ${ }^{2}$, Maciej K. Ginalski ${ }^{3}$, Andrzej J. Nowak ${ }^{4}$ and Luiz C. Wrobel ${ }^{5}$ \\ ${ }^{1}$ MMI Engineering Bristol \\ aduda@mmiengineering.com \\ ${ }^{2}$ Centre for CFD, University of Leeds \\ D.B.Ingham@leeds.ac.uk \\ ${ }^{3}$ ANSYS UK \\ maciej.ginalski@ansys.com \\ ${ }^{4}$ Silesian University of Technology \\ andrzej.j.nowak@polsl.pl \\ ${ }^{5}$ Brunel University \\ Luiz.Wrobel@brunel.ac.uk
}

\begin{abstract}
This paper presents numerical calculations of the temperature field obtained for the case of a neonate placed under a radiant warmer. The results of the simulations show a very non-uniform temperature distribution on the skin of the neonate, which may cause increased evaporation leading to severe dehydration. For this reason, we propose some modifications on the geometry and operation of the radiant warmer, in order to make the temperature distribution more uniform and prevent the high temperature gradients observed on the surface of the neonate. It is concluded that placing a high conductivity blanket over the neonate and introducing additional screens along the side of the mattress, thus recovering the radiation heat escaping through the side boundaries, helped providing more uniform temperature fields.
\end{abstract}

Key Words: Computational Fluid Dynamics, Heat Transfer, Natural Convection, Radiant Warmer, Neonatology

\section{Introduction}

Survival of each neonate depends on its ability to regulate its body temperature. While healthy and full-term babies usually have no difficulties in such adaptation, preterm and small neonates often cannot respond to environmental temperature changes. This is due to the immaturity of their thermoregulation system. Without an environment in which a preterm neonate can maintain a normal body temperature $\left(\sim 37^{\circ} \mathrm{C}\right)[1]$, it will risk cold stress and hypothermia, which may cause an increase in their morbidity and mortality [2], [3]. This can develop very quickly because a premature baby can receive up to $120 \mathrm{kcal} \mathrm{kg}^{-1} \mathrm{day}^{-1}$ [4], [5] and a wet newborn at birth looses around $200 \mathrm{kcal} \mathrm{kg}^{-1} \mathrm{~min}^{-1}$ [5]. For this reason, maintenance of the neonate's body within a narrow temperature range is essential for their survival and growth. Neonates nursed in an incubator, or under a radiant warmer within the thermo neutral zone, have also shown a more rapid weight gain in comparison to other babies. 
The importance of maintaining a newborn within a narrow temperature range is therefore crucial. However, there is one major concern when using radiant warmers, in that neonates may become severely dehydrated when nursed in these devices. Therefore, the main purpose of this paper is to present a numerical model of a neonate placed under a radiant warmer, which includes metabolic heat generation, and propose some modifications to improve the operation of the device.

The results presented in this paper were obtained using 3D numerical simulations performed in ANSYS FLUENT, for a simplified geometry of the neonate, namely when the newborn is modelled in the form of a half-cylinder, as well as for the real neonate geometry. Lastly, two different modifications are introduced to the shape and operation of the radiant warmer. They include placing a high conductivity blanket over the neonate and introducing additional screens along the sides of the mattress in order to recover the radiation that would otherwise escape through the side boundaries, and to re-direct this radiation towards the sides of the newborn.

\section{Assumptions}

The following assumptions have been made in the models described in this paper:

- The geometry and dimensions of the IW930 Series CosyCot ${ }^{\mathrm{TM}}$ Infant Warmer (Fisher \& Paykel, New Zealand) were used to build the model. This is because this type of radiant warmer was used when gathering the experimental data in the form of thermographic pictures.

- In some of the calculations, a simplified model of the neonate was used, namely a half-cylinder with the same volume as in the real neonate geometry.

- In the simplified model with a half-cylinder, only a quarter of the geometry is considered due to symmetry. In the model with the real geometry of the neonate, no symmetry planes are assumed.

- The radiant warmer is modelled in the form of a cylindrical filament with a prescribed heat flux. It was assumed that the known power of the radiant warmer is totally transformed into heat, and therefore the total heat flux on the external surface of the filament is equal to the power of the radiant lamp.

- The problem is modelled as steady-state.

- Turbulent flow is assumed and the Reynolds Averaged Navier-Stokes equations are used. The k- $\varepsilon$ RNG model is used for the turbulence modelling. This is because the model is effective for both high and low Re number turbulent flows and therefore it can produce good quality results even for the situations when the flow is not fully turbulent [6].

- The air density is calculated from the thermal equation of state for an ideal gas. The influence of pressure changes on the calculation of the density was assumed to be negligible in the considered situation, and the density was treated to be solely temperature dependent.

- Except for the density, all other air properties are assumed to be constant.

- The gravitational force is considered as a crucial part of natural convection.

- Radiation is taken into account. The Discrete Ordinates Model (DOM) is used for the radiation modelling because it is the only model in FLUENT that is able of calculating 
the specular reflection of the radiation occurring on the reflector. Furthermore, DOM is also a very reliable technique for modelling radiative heat transfer.

\section{Geometrical and material properties of the neonate's body}

For the purposes of this investigation, the body of the newborn is considered to be one solid material, and therefore all the material properties prescribed to it are averaged values based on information found in the literature.

\subsection{Volume and surface area}

From the geometry of a real neonate it was found that the volume of the newborn is approximately $0.002 \mathrm{~m}^{3}$, and its length is about $40 \mathrm{~cm}$. Therefore the same length was used to construct the geometry of the half-cylinder, for which the radius was worked out in such a way that the volume of the half-cylinder is the same as the volume of the real neonate. Therefore, for a cylinder of radius equal to $0.0573 \mathrm{~m}$ and length $40 \mathrm{~cm}$, the surface area of the half-cylinder is $0.128 \mathrm{~m}^{2}$. The surface area of the real neonate model is $0.146 \mathrm{~m}^{2}$.

\subsection{Mass of the newborn}

Three different correlations that relate the surface area of the newborn's body to its mass were employed to work out the mass of the neonate for its given size. Firstly, a linear correlation between the surface area of a neonate and its weight was derived based on two studies [7] and [8] performed on a group of newborns:

$$
m=14.961 \cdot A-0.4606
$$

where $m$ is the mass of the newborn, $\mathrm{kg}$, and $A$ is the surface area of the newborn, $\mathrm{m}^{2}$. Using Equation (1), the mass obtained for the surface area $A=0.146 \mathrm{~m}^{2}$ is $m=1.724 \mathrm{~kg}$. The second correlation was found in [9] and is given as follows:

$$
A=0.1 \cdot m^{2 / 3},
$$

and the third correlation from [8] is given by:

$$
A=m^{0.5378} \cdot h^{0.3964} \cdot 0.024265 \text {, }
$$

where $h$ is the newborn's height, $\mathrm{cm}$.

The surface areas of the newborn's body, for a given mass $m=1.724 \mathrm{~kg}$, calculated from Equation (2) and Equation (3), are $A=0.144 \mathrm{~m}^{2}$ and $A=0.140 \mathrm{~m}^{2}$, respectively. This shows good general agreement between the three methods used and gives us confidence that the obtained mass of the newborn, $m=1.724 \mathrm{~kg}$, is accurate enough. 
It has also been found in [10] that for low birth weight neonates, with a body mass of about $1.5 \mathrm{~kg}$, the average surface area of the body is $0.13 \mathrm{~m}^{2}$. This also confirms that the calculated weight $m=1.724 \mathrm{~kg}$ of the neonate in the presented model is realistic.

\subsection{Body tissue conductivity}

The average conductivity of the newborn's body has been found in [11] and it is $k=0.34 \mathrm{~W}$ $\mathrm{m}^{-1} \mathrm{~K}^{-1}$.

\subsection{Metabolic heat generation}

The metabolic heat generation was derived from a formula by Brück [12], which takes the following form:

$$
q_{v}=\frac{m\left(0.0522 \cdot \tau_{n}+1.64\right)}{V}
$$

where $q_{v}$ is the metabolic heat generation rate, $\mathrm{W} \mathrm{m}^{-3}$, and $\tau_{n}$ is the age of the newborn, days.

Taking the values of the newborn's mass $m$ and volume $V$ presented above, as well as assuming that the neonate is, say, one day old, the metabolic heat generation rate obtained is $q_{v}=1417.5 \mathrm{~W} \mathrm{~m}^{-3}$, which gives $Q=2.9 \mathrm{~W}$. This value is consistent with the findings presented in [7], where a newborn weighing $1.6 \mathrm{~kg}$ had a metabolic heat production of about $2.8 \mathrm{~W}$.

\section{Geometry, mesh and boundary conditions}

\subsection{Simplified geometry of a neonate}

The geometry used for the initial simulations described in this paper is presented in Figure 1. The length of the half-cylinder was set to be $0.4 \mathrm{~m}$, since this is the height of the newborn in the real model, and its radius was taken to be $0.0573 \mathrm{~m}$. With such dimensions, the volume of the half-cylinder is equal to the one of a real neonate.

The mesh size used to prepare the grid inside the half-cylinder is $0.008 \mathrm{~m}$, which results in a mesh of $6.5 \mathrm{k}$ elements. The remainder of the domain consists of $258 \mathrm{k}$ elements.

The boundary conditions used in the model are presented in Figure 2. The side and top boundaries of the model are pressure inlets and a pressure outlet with the air temperature of $22{ }^{\circ} \mathrm{C}$ and no gauge pressure prescribed. This is the temperature used in the nursing rooms for neonates where no intensive care is necessary. The temperature in intensive care units is usually about $27^{\circ} \mathrm{C}$. The external blackbody temperature of $20^{\circ} \mathrm{C}$ is also prescribed at the inlet and outlet boundaries. By prescribing the external blackbody temperature at the inlets and the outlet, we can take into account the radiation heat losses from such surfaces as the mattress and cylinder to the colder walls of the room. 


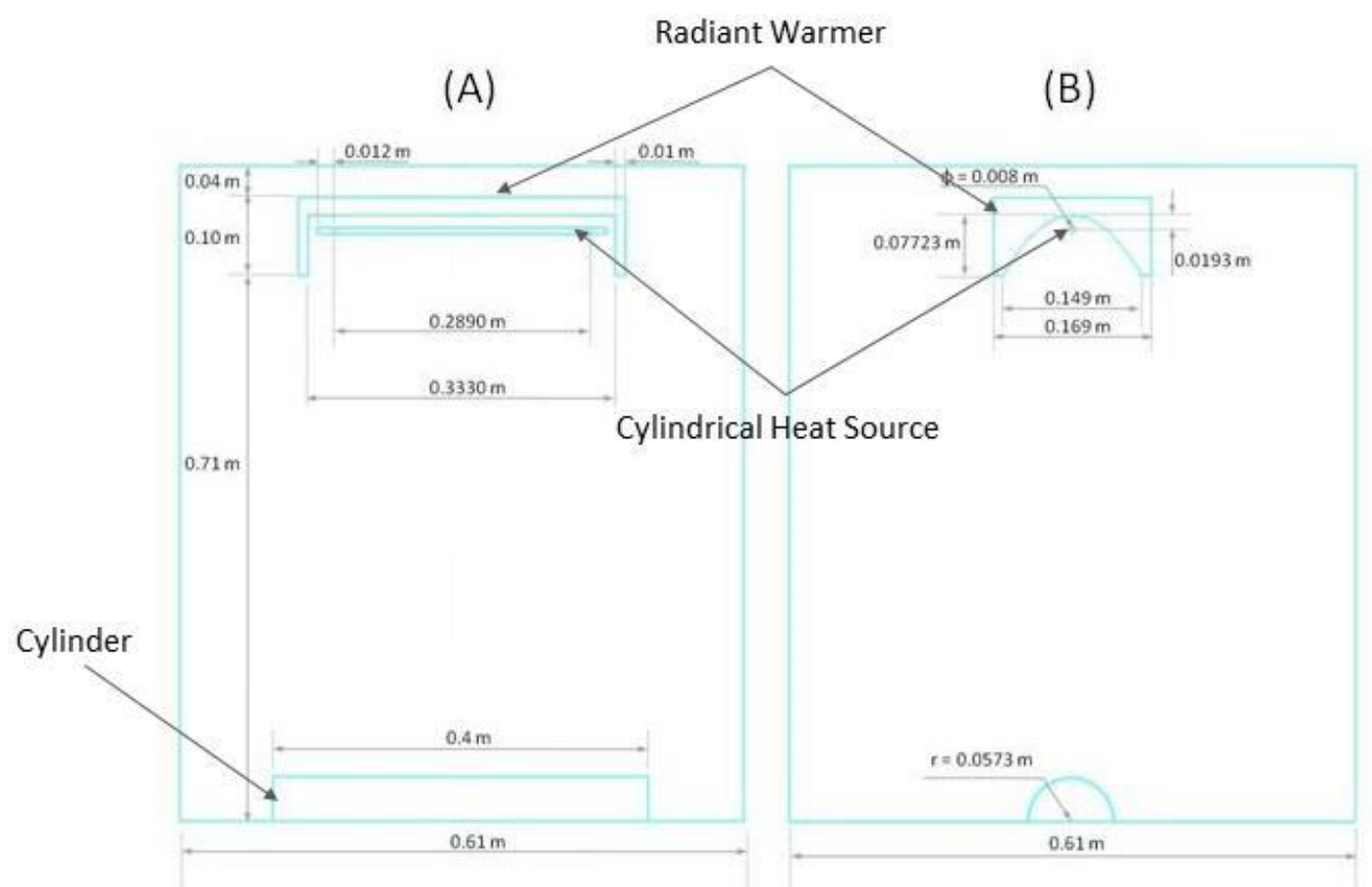

Figure 1 The dimensions of the computational domain: side view (A), front view (B) of the model.

(A)

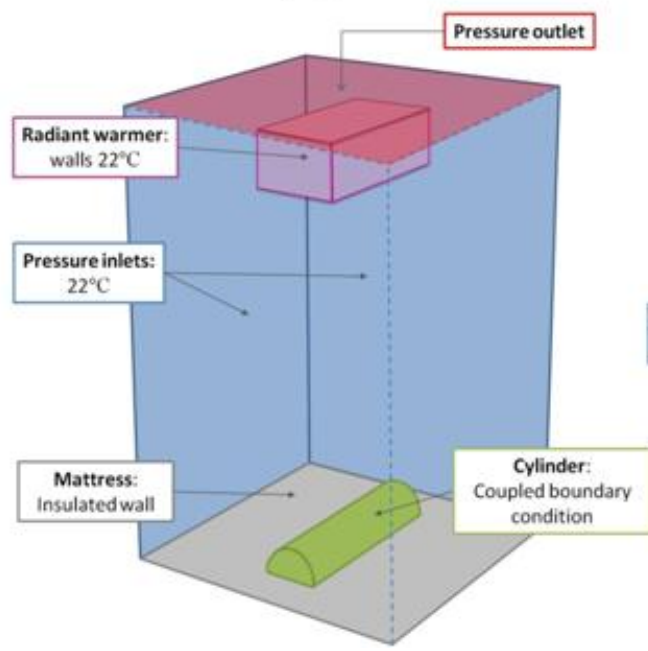

(B)

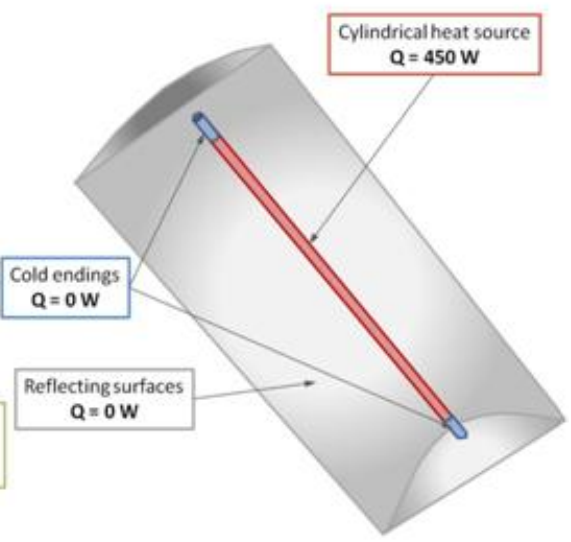

Figure 2 The boundary conditions prescribed: $(A)$ a general view of the computational domain, and (B) a bottom view of the radiant lamp.

The mattress is assumed to be an insulated wall and the cylinder has a coupled boundary condition prescribed which is a continuity boundary condition. This was possible because the surface of the cylinder lies on the interface between the solid (the inside of the cylinder modelling the human body) and the fluid (air). This boundary condition simply means that the amount of heat reaching the surface of the cylinder, from the side of the solid, has to be transferred directly to the air. This implies that there is no heat accumulation at the surface of the half-cylinder. The bottom of the half-cylinder is assumed to be insulated. The volume of the half-cylinder has a metabolic heat production prescribed in the form of a volumetric heat source equal to $1417.5 \mathrm{~W} \mathrm{~m}^{-3}$. 
The last part of the model is the radiant lamp, which is placed $71 \mathrm{~cm}$ above the mattress. The side and top walls of the radiant warmer have a temperature boundary condition prescribed at $22{ }^{\circ} \mathrm{C}$. The inside of the lamp consists of an insulated reflector and a filament, in which the sides are insulated and the central part is the actual heat source with a power of $450 \mathrm{~W}$.

The values of the internal emissivity and the diffuse fraction for the reflector and the heat source surfaces were obtained from the manufacturer. The emissivity of the reflector is set to be 0.04 and the emissivity of the heat source is 0.95 . Diffuse fractions of 0.05 and 0.95 have been prescribed to the reflector and the heat source, respectively.

\subsection{Real geometry of a neonate}

In this model, all the boundary conditions are as presented in Figure 2.

TGrid was used to generate the mesh, see Figure 3, and the whole model contains $183 \mathrm{k}$ tetrahedral elements.

In this study, the baby's legs are separated and the arms are outstretched. In reality, ill babies are often nursed with their legs next to each other, and their arms are often by their sides. However, the presented approach was considered conservative, because the outstretched arms and separated legs would lose more heat and absorb radiant heat differently than if held close to the body.

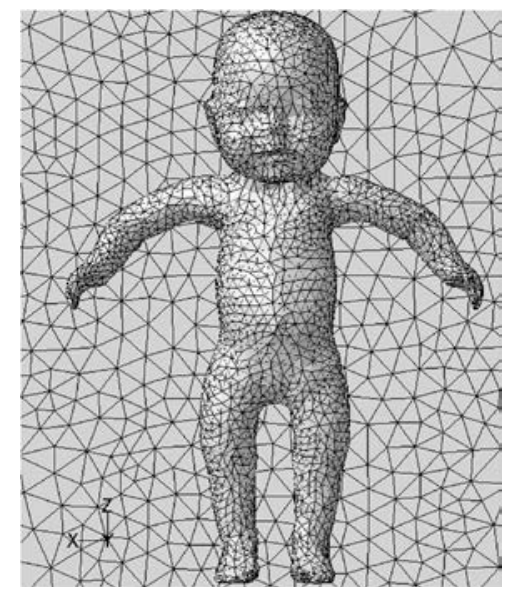

Figure 3 The mesh constructed on the neonate and the mattress, seen from the top.

\section{Results}

All the results presented in this paper were obtained using ANSYS FLUENT v12.

The temperature fields obtained on the mattress and the half-cylinder are presented in Figure 4. It can be observed that the temperatures on the mattress are higher than the temperatures obtained on the half-cylinder. This is due to the fact that in the locations on the mattress next to the half-cylinder, the convective heat losses are hindered by the presence of the half-cylinder. 


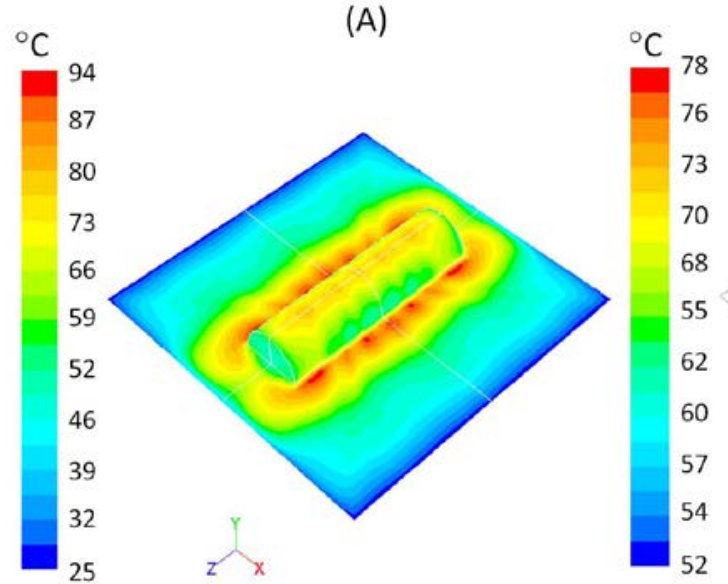

(B)

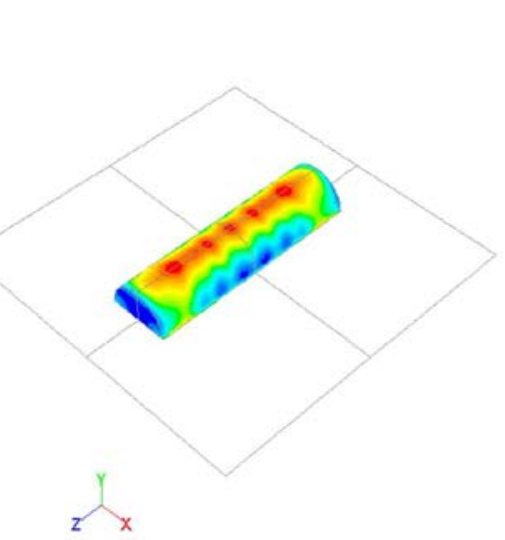

Figure 4 The temperatures on: (A) mattress and half-cylinder, and (B) half-cylinder.

The temperatures on the skin of the newborn obtained for the real neonate geometry are presented in Figure 5. It can be observed that the temperatures obtained on the skin of the neonate are higher on the upper parts of the body and much lower at the sides, which are not directly warmed by the radiant heat source. This is in agreement with the results obtained for the simplified neonate geometry.

Such high temperature gradients and non-uniform temperature fields may lead to enhanced evaporation from the newborn. It is possible that this situation could be partly avoided if the temperatures obtained on the newborn were more uniform.

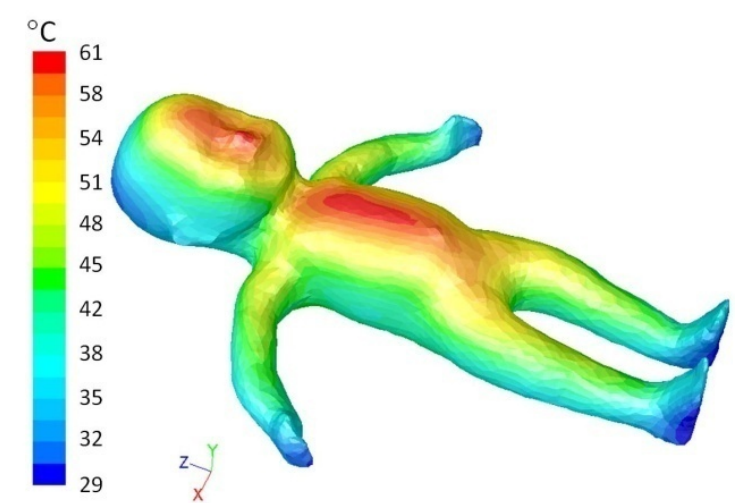

Figure 5 The temperatures on the skin of the newborn obtained for the real geometry of the neonate.

\section{Modifications to the operation of a radiant warmer}

The results presented so far in this paper show a very non-uniform temperature distribution on both the half-cylinder and the real geometry of the neonate nursed under the radiant warmer. This is due to the large amount of radiation received by the top part of the body, leaving the sides significantly colder. Such significant temperature gradients should be avoided because they are considered one of the reasons for the dehydration of neonates nursed under radiant warmers. For these reasons, in this part of the paper we focus on 
finding a solution for the problem of the high temperature gradients obtained on the surface of the newborn.

\subsection{Blanket around the cylinder}

The purpose of this investigation is to determine whether placing a blanket of high thermal conductivity around the newborn will assist in preventing the creation of high temperature gradients on the surface of the newborn nursed under a radiant warmer. Therefore, the influence of placing a blanket over the half-cylinder was investigated. The thickness of the blanket was assumed to be $1 \mathrm{~cm}$.

Two different values of the thermal conductivity have been considered, namely $0.033 \mathrm{~W} \mathrm{~m}^{-1}$ $\mathrm{K}^{-1}$, being a typical value of the thermal conductivity of a wool blanket, and $20 \mathrm{~W} \mathrm{~m}^{-1} \mathrm{~K}^{-1}$, in order to investigate two relatively extreme values of the thermal conductivity. The resulting temperature fields obtained on the half-cylinder after covering it with blankets of the two mentioned thermal conductivities are presented in Figure 6. It should be noted that two different temperature scales were used for presenting the results in Figure 6.

(A)

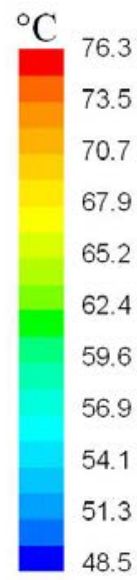

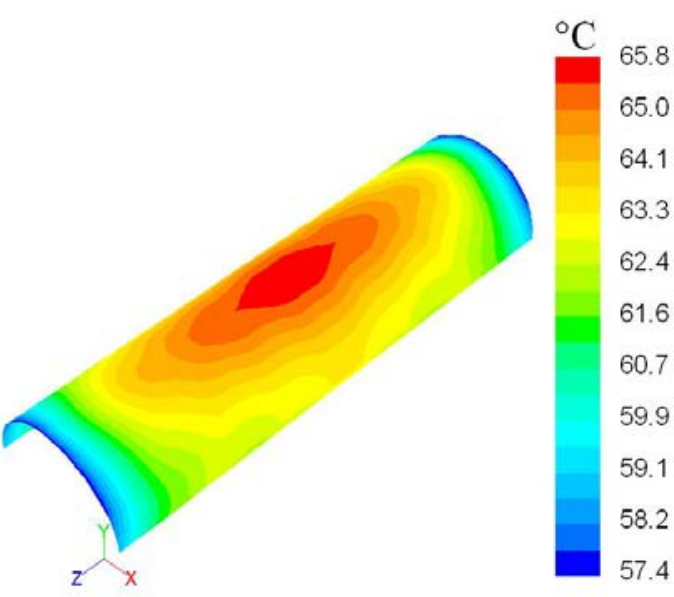

(B)

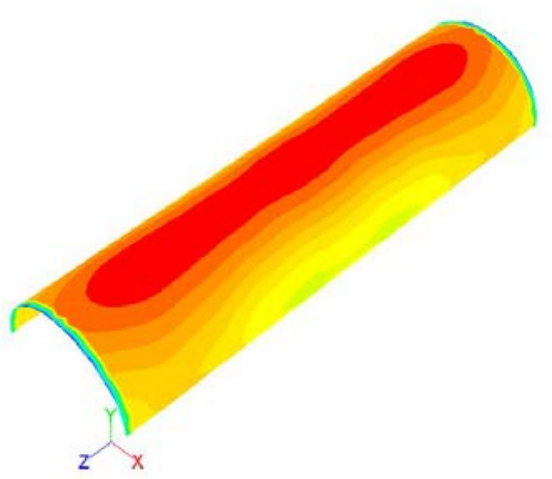

Figure 6 The temperature fields obtained on the half-cylinder covered with a blanket of the following thermal conductivities: (A) $0.033 \mathrm{~W} \mathrm{~m}^{-1} \mathrm{~K}^{-1}$, and (B) $20 \mathrm{~W} \mathrm{~m}^{-1} \mathrm{~K}^{-1}$.

It can be observed from Figure $6(\mathrm{~A})$ that even a blanket with a small conductivity, namely $0.033 \mathrm{~W} \mathrm{~m}^{-1} \mathrm{~K}^{-1}$, had a positive impact on the temperature gradients obtained on the surface of the half-cylinder. This is due to the fact that the temperature difference between the top and sides of the half-cylinder decreased from about $25^{\circ} \mathrm{C}$ for the situation without the blanket, see Figure 4 (B), to about $10^{\circ} \mathrm{C}$. Moreover, covering the neonate with a blanket of higher conductivity, namely $20 \mathrm{~W} \mathrm{~m}^{-1} \mathrm{~K}^{-1}$, resulted in a further improvement in the temperature gradients on the newborn, and it can be concluded from Figure 6 (B) that the temperature gradients between the top and the sides of the half-cylinder reduced by a further $4^{\circ} \mathrm{C}$.

It can be observed from Figure 6 that for the situation with the higher thermal conductivity blanket, the maximum temperatures obtained on the surface of the newborn were reduced by more than $10{ }^{\circ} \mathrm{C}$ when compared to the situation with a lower thermal conductivity and without a blanket. The blanket with the thermal conductivity equal to $20 \mathrm{~W} \mathrm{~m}^{-1} \mathrm{~K}^{-1}$ 
created a more uniform temperature field on the half-cylinder. Moreover, no significant change has been observed in the average surface temperatures on the half-cylinder for the cases compared in Figure 6.

In addition, it should be noted that in the described simulations with a blanket placed on top of the half-cylinder, perfect contact is assumed at the interface between the blanket and the baby. In reality, the blanket will not be in perfect contact with the newborn's skin, as there will always be a layer of air between them. However, the thermal conductivity of air is about $0.025 \mathrm{~W} \mathrm{~m}^{-1} \mathrm{~K}^{-1}$, and this is of a similar magnitude as one of the considered thermal conductivities of the blanket, namely $0.033 \mathrm{~W} \mathrm{~m}^{-1} \mathrm{~K}^{-1}$. Because the low conductivity blanket also helped in creating a more uniform temperature field on the half-cylinder, it can be concluded that the layer of air between the blanket and the skin of the newborn will have a similar impact on the results. Adding to this the effect of placing a high conductivity blanket over the baby, the temperature gradients obtained on the surface of the newborn will also decrease, even with the layer of air present between the blanket and the half-cylinder.

\subsection{Screens around the mattress}

In this section, a modification to the geometry of the radiant warmer is introduced as additional reflecting screens are placed at the sides of the mattress, which direct all the radiation that would otherwise escape through the sides of the domain towards the newborn. In Figure 7, the radiation emitted from the heat source was divided into three main sectors:

- blue - the part of the radiation that is not reflected from the reflector and reaches the mattress, 12\%;

- green - the part of the radiation that is not reflected from the reflector and escapes through the side boundaries of the computational domain, 17\%;

- red - the part of the radiation that is reflected from the reflector and directed towards the newborn, $71 \%$.

It can be observed from Figure 7 that about $17 \%$ of the total radiation emitted from the heat source is being lost through the side boundaries of the computational domain. These losses can be easily recovered by installing additional reflecting screens at each side of the mattress. The height of these screens is determined by the angle of the last ray leaving the heat source which does not reach the reflector, and this is illustrated by the black arrow in Figure 7. 


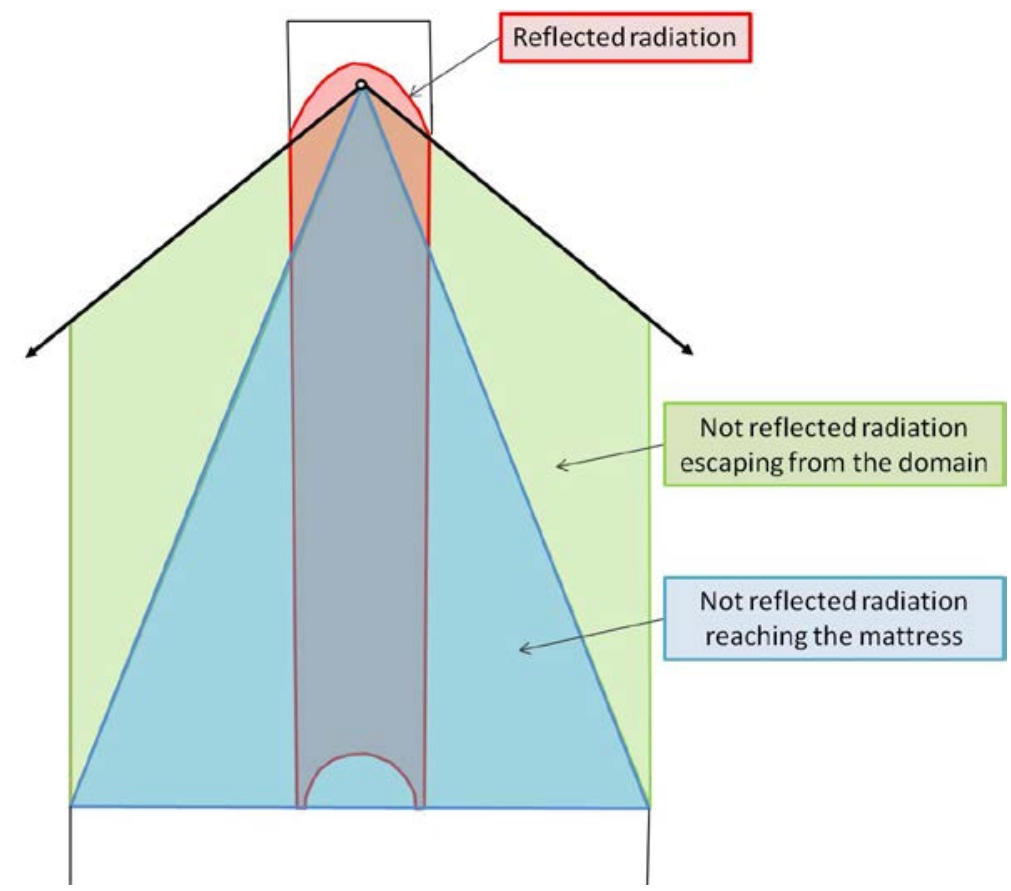

Figure 7 An illustration of the different sections where the radiation emitted from the heat source can be directed.

When using side screens, we can make sure that all of the radiation that would normally escape from the domain will now be redirected towards the mattress and used to warm up the sides of the newborn. A ray tracking program was developed for the purpose of finding the best possible shape of the screens, and directing the recovered radiation towards the sides the half-cylinder. The top of the cylinder is already heated enough by the radiation coming directly from the radiant lamp, therefore there is no need to direct the recovered radiation to this vicinity. Instead, the design of the side screens is aimed at warming up the sides of the cylinder, which will lead to decreasing the temperature gradients on the surface of the newborn. After a series of tests, the optimal parabolic shape of the screens was determined, which has been defined by the coordinates of the points presented in Table 1 , where point 1 is the bottom point lying on the mattress and point 3 is the highest point of the screen.

A 3D CFD model with the screens located along the sides of the mattress was built. In order to incorporate the presence of the screens into the model, the computational domain was extended in both the positive and negative $x$ directions, i.e. horizontally across the width of the half-cylinder, by a distance of $0.3 \mathrm{~m}$. A pressure inlet boundary condition was prescribed to the two new boundaries introduced at the bottom.

Table 1 Coordinates of the points used to create both screens.

\begin{tabular}{|c|c|c|c|}
\hline Point No & $\boldsymbol{x}_{\text {left }} \mathbf{m}$ & $\boldsymbol{x}_{\text {right }}, \mathbf{m}$ & $\boldsymbol{y}, \mathbf{m}$ \\
\hline 1 & -0.305 & 0.305 & 0.787 \\
2 & -0.404 & 0.404 & 0.553 \\
3 & -0.414 & 0.414 & 0.339 \\
\hline
\end{tabular}


For the purpose of the radiation calculations, it was assumed that the screens are made from the same material as the reflector, and therefore the internal emissivity is 0.04 and the diffuse fraction is 0.05 . The remaining boundary conditions, material properties and model settings are as presented in Section 4 of this paper. The mesh constructed for the presented geometry consists of $532 \mathrm{k}$ tetrahedral and wedge-shaped elements.

The temperature fields obtained on the mattress and the half-cylinder for the situation with the screens is presented in Figure 8. It can be observed that after including the screens into the model, the temperatures obtained on the mattress increased by about $10{ }^{\circ} \mathrm{C}$ as more radiation is now directed towards the mattress. The presence of the screens provides a more enclosed environment, which leads to increased air temperatures close to the newborn and the mattress.

In order to more accurately compare the temperatures on the half-cylinder, several lines along the circumference of the cylinder were created for different locations in the $z$ direction, i.e. along the length of the cylinder, which are illustrated in Figure 8. A comparison of the temperature plots for these locations, for the situations both with and without the side screens, is presented in Figure 9. It should be noted that the position $x=0$ $\mathrm{m}$ on the discussed plots represents the very top of the half-cylinder, while $x=0.06 \mathrm{~m}$ and $x=-0.06 \mathrm{~m}$ are the side ends of the half-cylinder.

It can be observed from Figure 9 that the temperature difference on the half-cylinder decreased from about $25{ }^{\circ} \mathrm{C}$, for the situation without the screens, to about $12{ }^{\circ} \mathrm{C}$ after including the screens in the model. This shows a significant improvement in the temperatures obtained on the half-cylinder over the situation without the side screens.

It should be noted that the investigation on the impact of the screens was performed for the simplified geometry of the half-cylinder, however the screens would work equally as well for the real geometry of the newborn. This is due to the fact that the diameter of the halfcylinder, being about $11 \mathrm{~cm}$, and the width of the trunk and the head of the real newborn geometry are approximately the same as the diameter of the half-cylinder. This means that if the radiation recovered by the screens was directed towards the sides of the half-cylinder, it would similarly be directed towards the sides of the newborn, thus warming them up and decreasing the unfavourable large temperature gradients on the surface of the neonate. 


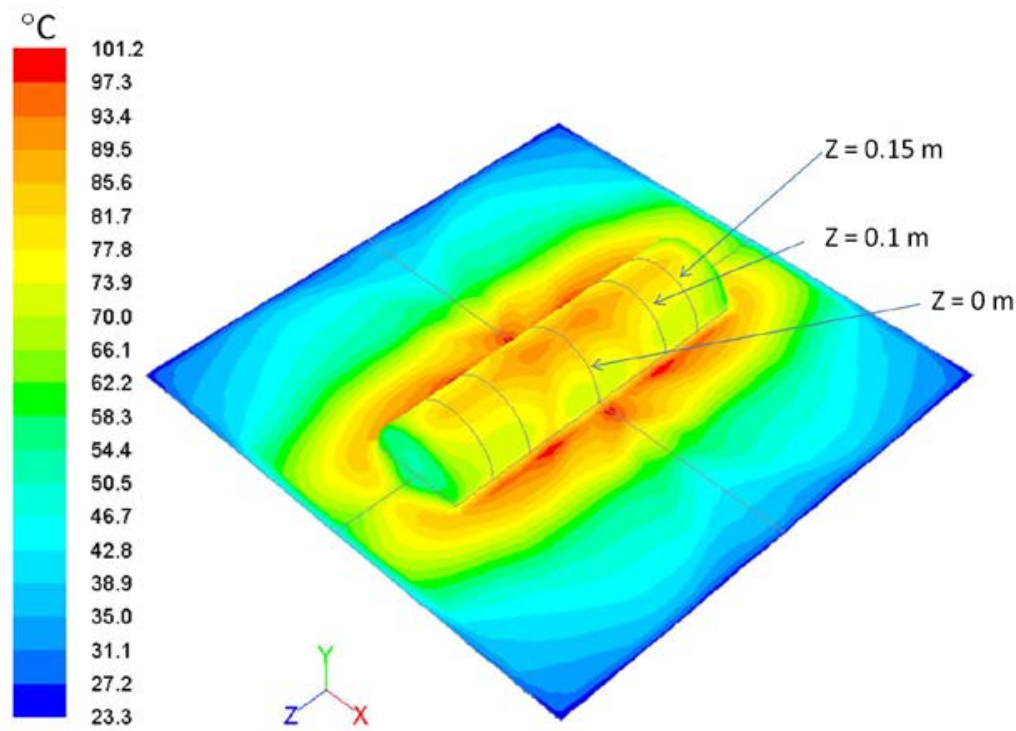

Figure 8 The temperature fields obtained on the mattress and the cylinder for the situation when the side screens are placed at both sides of the mattress and the position of the lines along the circumference of the cylinder used to create the temperature plots.

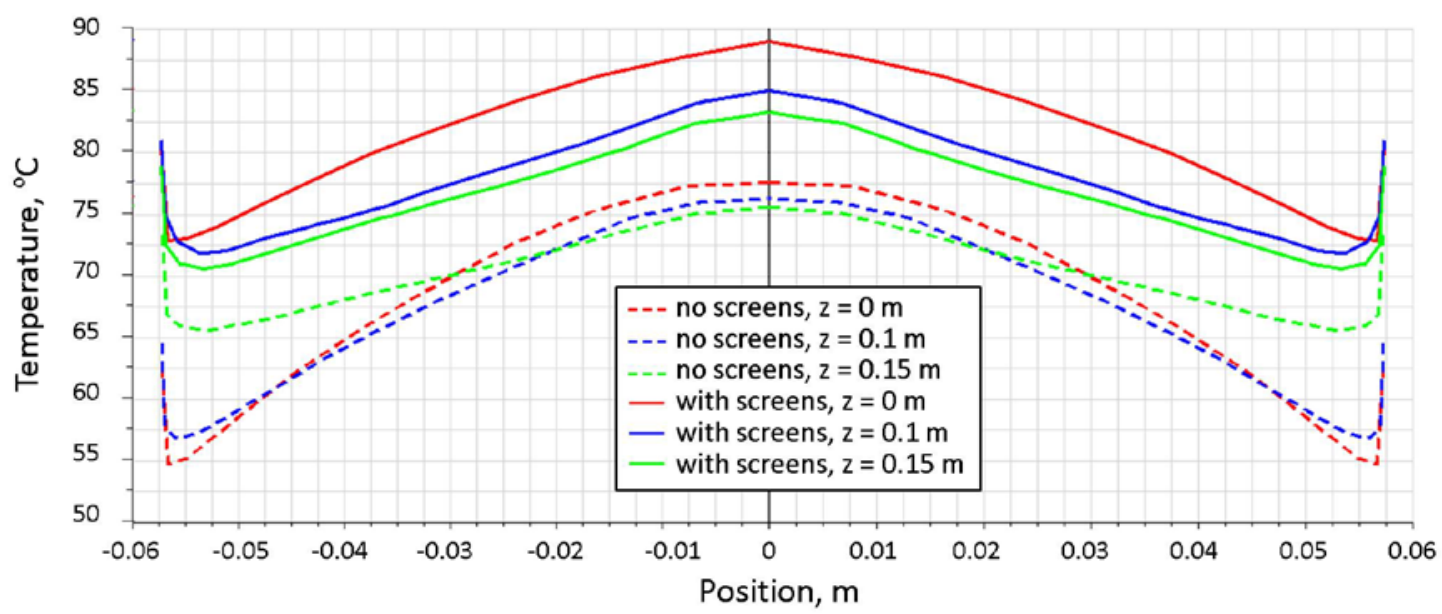

Figure 9 Comparison of the temperature plots obtained on the surface of the cylinder for different $z$ locations along the length of the cylinder, for the situations both with and without the side screens.

\section{Conclusions}

In this paper, we have presented numerical results for the temperature distribution of a moderately premature neonate nursed under a radiant warmer. For simplicity, the neonate is considered to be healthy and the composition and skin thickness of different body areas are considered to be constant. The heat loss of a neonate with some illness, e.g. a severe infection, would be higher due to vasodilatation, but this case is not considered in this paper.

The results presented in the first part of the paper show that the newborn nursed under the radiant warmer suffers from a very non-uniform skin temperature distribution, where the 
top regions of the body are much warmer than the sides of the neonate. This problem of some parts on the body becoming overheated is connected to increased water losses and may cause dehydration. Therefore, in the second part of this paper, we have focused on finding possible solutions for improving the temperature distribution on the newborn and preventing the high temperature gradients obtained on the surface of the neonate.

Firstly, the influence of placing a blanket over the newborn was investigated. In clinical practice, babies nursed under radiant warmers are rarely covered, and this is due to the need for constant observation of sick neonates, as well as fear that blankets will reduce the heat received by the baby or make temperature monitoring more difficult. However, it has been found that a high conductivity blanket significantly decreases the temperature gradients over the whole surface and this creates a much more uniform temperature distribution on the newborn. It has also been discovered that even a blanket with insulating thermal properties assists in shielding the newborn from the direct influence of the radiant lamp, and helps spreading the heat over the whole body surface. This is a very important finding because it illustrates that a simple change, such as the presence of a blanket, could substantially improve the comfort of the neonate nursed under a radiant warmer.

Next, additional screens were introduced at the sides of the mattress in the direction along the axis of the half-cylinder. It was found that after introducing the side screens, a significant reduction in the temperature gradient obtained on the body surface could be observed, as the temperatures of the sides of the body increased due to the additional radiation directed into this vicinity by the screens. This is a very important finding and it means that a significant improvement in the temperature distribution on the newborn nursed under a radiant warmer can be obtained by a simple modification to the radiant warmer. Moreover, the screens are only placed by the sides of the mattress along the sides of the baby, which leaves the parts of the mattress in the vicinity of the head and the feet of the newborn open to access by the nursing staff. It has also been observed that the screens shield the neonate from the radiation losses to the colder walls of the room, providing an additional advantage of being able to decrease the power of the radiant warmer to keep the newborn warm.

Both solutions proposed in this paper, fulfil the goal of creating a more uniform temperature distribution over the surface of the newborn and this is thought to decrease the possibility of dehydration of neonates nursed under radiant warmers. The first proposed method, including placing a blanket around the baby, preferably of a high heat conductivity, can be implemented by the staff of the neonatal unit. The second solution suggested in this paper includes placing additional screens at the sides of the mattress and this needs to be performed by the radiant warmer manufacturers.

\section{Acknowledgements}

We would like to express our appreciation to the European Union for the Marie Curie Fellowship grant awarded to the Centre for CFD, University of Leeds. We would also like to acknowledge Prof Denis Azzopardi from Hammersmith Hospital, London, and Dr Gabriel Wecel from Silesian University of Technology for their support on this project. The authors also greatly appreciate the help of Fisher \& Paykel in supplying the necessary data for the calculations. 


\section{Bibliography}

[1] G. Boxwell, Neonatal Intensive Care Nursing, Chapter 4: London: Routledge , 2000.

[2] R. Knobel and D. Holditch-Davis, "Thermoregulation and heat loss prevention after birth during neonatal intensive care unit stabilization of extremely low-birth weight infants," Journal of Obstetric, Gynecologic and Neonatal Nursing, vol. 36, pp. 280-287, 2007.

[3] A. Lyon, "Temperature control in the newborn infant," Current Paediatrics, vol. 16, pp. 386-392, 2006.

[4] C. V. Hulzebos and P. J. J. Sauer, "Energy requirements," Seminars in Fetal and Neonatal Medicine, vol. 12, pp. 2-10, 2007.

[5] C. D. Nalepka, "Understanding thermoregulation in newborns," Journal of Obstetric, Gynecologic and Neonatal Nursing, vol. 5, pp. 17-19, 1976.

[6] F. Boysan, "Advanced Turbulence Modelling; Short Course", Ansys FLUENT.

[7] S. Baumgart, "Partitioning of heat losses and gains in premature newborn infants under radiant warmers," Pediatrics, vol. 75, pp. 89-99, 1985.

[8] S. Baumgart, W. D. Engle, W. W. Fox and R. A. Polin, "Radiant warmer power and body size as determinants of insensible water loss in critically ill neonate," Pediatric Research, vol. 15, pp. 1495-1499, 1981.

[9] J. R. Hill and K. A. Rahimtulla, "Heat balance and the metabolic rate of new-born babies in relation to environmental temperature; and the effect of age and weight on basal metabolic rate," The Journal of Physiology, vol. 180, pp. 239-265, 1965.

[10] G. B. Avery, M. G. MacDonald, M. M. K. Seshia and M. D. Mullett, Avery's Neonatology: Pathophysiology \& Management of the Newborn, Philadelphia: Lippincott Williams \& Wilkins, 2005.

[11] M. K. Ginalski, "Numerical analysis of heat and mass transfer processes within an infant incubator," Ph.D. Thesis. Silesian University of Technology, Institute of Thermal Technology, Gliwice, Poland, 2007.

[12] K. Bruck, "Temperature regulation in the newborn infant," Neonatology, vol. 3, pp. 65-119, 1961.

[13] M. K. Ginalski, A. J. Nowak and L. C. Wrobel, "Modelling of heat and mass transfer processes in 
neonatology," Biomedical Materials, vol. 3, Paper 034113, 2008.

[14] G. Wecel, Z. Ostrowski and R. A. Bialecki, "A novel approach of evaluating absorption line black body distribution function employing proper orthogonal decomposition," Journal of Quantitative Spectroscopy \& Radiative Transfer, vol. 111, pp. 309-317, 2010.

[15] L. C. Wrobel, M. K. Ginalski, A. J. Nowak, D. B. Ingham and A. M. Fic, "An overview of recent applications of computational modeling in neonatology," Philosophical Transactions of the Royal Society, Series A: Mathematical, Physical and Engineering Sciences, vol. 368, pp. 28172834, 2010.

[16] A. M. Fic, D. B. Ingham, M. K. Ginalski, A. J. Nowak and L. C. Wrobel, "Heat and mass transfer under an infant radiant warmer--development of a numerical model," Medical Engineering and Physics, vol. 32, pp. 497-504, 2010. 